\title{
OTIMIZAÇÃO DE CORTE PARA EMBALAGENS: UM ESTUDO DE CASO
}

\author{
Rafaela Boeira Cechin (Universidade de Caxias do Sul - UCS) rbcechin@ucs.br \\ Bruna Caroline Orlandin (Universidade De Caxias Do Sul - UCS) bcorlandin@ucs.br \\ Nathalia Tessari Moraes (Universidade De Caxias Do Sul - UCS) ntmoraes1@ucs.br \\ Bruna Gabriele de Matos (Universidade De Caxias Do Sul - UCS) bgmatos@ucs.br \\ Leandro Corso (Universidade de Caxias do Sul - UCS) llcorso@ucs.br
}

\begin{abstract}
Resumo
Evitar desperdício de matéria-prima possibilita redução de custos, o que permite melhorar a margem de lucro da empresa, servindo como incentivo para otimizar os processos produtivos, tornando-os os mais eficientes possíveis. Para isso, estudos acadêmicos sobre métodos de otimização são essenciais para auxiliar gestores na tomada de decisões dentro das organizações. Este trabalho aplicou Programação Linear em problema de corte unidimensional de estoque, com o propósito de otimizar o processo de corte de matéria-prima. Para isso, foi desenvolvido um modelo matemático para otimizar este procedimento e foi coletado informações sobre a demanda e políticas da empresa presente na Serra Gaúcha, no estado do Rio Grande do Sul. Então, os resultados obtidos com o modelo matemático sugerido foram comparados com o atual cenário da organização e constatouse que é possível uma redução para $950 \mathrm{~cm}$ de matéria-prima desperdiçada, que representa uma diminuição de quase $40 \%$.
\end{abstract}

Palavras-Chaves: Programação Linear, Otimização, Problema de corte de estoque unidimensional, Redução de desperdício

\section{Introdução}

As organizações devem estar sempre preocupadas em tornar seus processos produtivos o mais eficiente possível, melhorando-os para poder aumentar a lucratividade e a competitividade, distinguindo-se dos concorrentes. Para isso, devem tirar proveito que os avanços computacionais e os estudos acadêmicos podem proporcionar.

Uma maneira de se destacar no mercado é tendo preço acessível, sem perder a qualidade dos produtos. Uma das possibilidades para reduzir custos é evitar desperdícios de matéria-prima durante o processo produtivo, viabilizando redução no custo de fabricação do produto, custo com compra de material excessivo e horas de trabalho. Trabalhos acadêmicos podem contribuir em exemplos como este, com a utilização de métodos de otimização, auxiliando na tomada de decisão para tornar o processo produtivo mais eficiente.

A área de Pesquisa Operacional (PO) possui diversas técnicas matemáticas de otimização que podem ser aplicadas em organizações para resolução de problemas, possibilitando novas soluções para dificuldades com alocação de recursos, redução de custos, entre outros. A 
multidisciplinaridade da destas técnicas permitem uma contribuição na tomada de decisões com aplicação em qualquer atividade (ANDRADE, 2015). Uma das ferramentas da PO é a Programação Linear (PL), em que é possível resolver diversos problemas de otimização, se todas as funções matemáticas forem lineares.

Segundo Almeida (2018), a grande relevância da PL pode ser justificada por ser uma importante técnica que possibilita aumento do lucro para a maioria das companhias nos países industrializados. Como é uma ferramenta eficiente para alocar recursos ilimitados, a sua aplicação está avançando para outros setores da sociedade, já que o resultado é a maximização da eficiência na alocação de recursos (PERINI et al., 2013).

Desta forma, objetiva-se encontrar a combinação ótima de produtos de forma a reduzir o descarte te matéria-prima por meio da aplicação de PL. O modelo matemático desenvolvido engloba uma aplicação de diferentes tamanhos de etiquetas a serem produzidas.

\section{Referencial teórico}

Nesta seção, é estudado os conceitos teóricos de PL e o Problema de Corte de Estoque.

\subsection{Programação linear}

A PL é um dos maiores avanços do século XX (HILLIER; LIEBERMAN, 2013). Desenvolvida após a Segunda Guerra Mundial e com o objetivo de auxiliar na alocação de recursos, a PL é uma das ferramentas de gestão mais utilizadas na determinação da política de estoques, em fluxos de caixa e investimentos, na organização de transportes, dentre outras aplicações (ANDRADE, 2015).

De acordo com Silva et al. (2017), a PL é amplamente utilizada devido a simplicidade de seus modelos e a possibilidade de programação de uma técnica de solução em computadores. Para Longaray (2013, p. 59), a PL é definida como uma "técnica cuja estrutura algébrica é a que mais se aproxima da forma geral matemática que os modelos de otimização preconizam”.

Um modelo é a representação de um objeto, processo real ou sistema elaborado por meio de estruturas matemáticas como funções, equações e matrizes. Estas estruturas são utilizadas para descrever e analisar as características do que está sendo modelado (CARTER; PRICE, 2001). 
Para formular um problema de otimização, é necessário identificar a função objetivo, as variáveis e as restrições do modelo (GOMES; CORSO, 2015). Moreira (2007) comenta que a função objetivo é a solução do problema, que pode ser de maximização ou minimização, as variáveis de decisão referem-se a grandezas que assumem diversos valores, os parâmetros são conhecidos como os coeficientes das variáveis de decisão e as restrições do modelo representam os limites sobre as ações executadas na função objetivo.

Considerando que um problema de PL pode ser representado por meio de uma função objetivo e de um conjunto de restrições, ambos lineares, nas Equações de 1 a 3 tem-se o modelo geral de PL (LOESCH; HEIN, 2009).

$$
\{\operatorname{Max}, \operatorname{Min}\} Z=c_{1} x_{1}+c_{2} x_{2}+\cdots+c_{n} x_{n}
$$

Sujeito a:

$$
\begin{gathered}
a_{11} x_{1}+a_{12} x_{2}+\cdots+a_{1 n} x_{n}\{=, \leq, \geq\} b_{1} \\
a_{21} x_{1}+a_{22} x_{2}+\cdots+a_{2 n} x_{n}\{=, \leq, \geq\} b_{2} \\
\vdots \\
a_{m 1} x_{1}+a_{m 2} x_{2}+\cdots+a_{m n} x_{n}\{=, \leq, \geq\} b_{m}
\end{gathered}
$$

Sendo:

$$
x_{1} \geq 0, x_{2} \geq 0, \cdots, x_{n} \geq 0
$$

Onde:

a) $x_{1}, x_{2}, \cdots, x_{n}$ são as variáveis estruturais do problema, escolhidas para que as restrições sejam satisfeitas e a função objetivo otimizada;

b) $c_{1}, c_{2}, \cdots, c_{\mathrm{n}}$ são coeficientes da função objetivo;

c) $a_{i j}$ e $b_{j}$ são coeficientes das restrições, ou seja, são os limites a serem considerados;

d) $(\{\operatorname{Min} Z\}=\cdots)$ ou $(\{\operatorname{Max} Z\}=\cdots)$ é a função objetivo, mostra a meta que se deseja alcançar, seja de minimização ou de maximização. 


\subsection{Problema de corte de estoque}

Ghidini (2009) e Poldi e Arenales (2010) explicam que problema de corte de estoque consiste em pegar matéria-prima disponível em estoque e produzir, com processo de corte, peças menores, de forma otimizada que enfatize na redução de custo ou de desperdício de material. Poldi (2003) e Alem Junior (2007) ainda comentam da crescente motivação para este tipo de estudo, por ser um problema importante devido a aplicabilidade econômica e aliada à complexidade computacional e de resolução da otimização combinatória.

Como geralmente há perda indesejável de matéria-prima, Farias (2011) e Assis (2019) ressaltam a importância de estudos de otimização, para minimizar o desperdício do material e consequentemente reduzir custos, ou ainda, maximizar a quantidade de peças cortadas, consumindo a matéria-prima de forma mais eficiente.

De acordo com Poldi (2003), Alem Junior (2007), Ghidini (2009) e Farias (2011), uma das classificações para problema de corte é relacionado com a dimensão relevante da peça, com as seguintes categorizações: problemas de corte unidimensional, bidimensional ou tridimensional. Este trabalho analisou um problema unidimensional, já que apenas uma dimensão da peça é relevante no processo de corte, sendo a dimensão do comprimento, da largura, ou da altura. Este tipo de problema é usualmente visto em indústrias de papel, chapas de madeira ou de aço, metalúrgicas, vidro, móveis, têxtil, plástica, entre outras (ALEM JUNIOR, 2007; POLDI; ARENALES, 2010).

Para a modelagem matemática de um problema de corte unidimensional, de uma forma geral, são necessárias duas etapas: definição de todos possíveis padrões de corte, e determinação da quantidade de vezes que cada padrão poderá ser usado. Farias (2011) e Assis (2019) explicam que a forma cortar o material em estoque denomina-se padrão de corte. Ainda, Poldi (2003) e Alem Junior (2007) comentam que as dificuldades computacionais relacionadas a este tipo de problema são resolvidas com a condição de integralidade das variáveis e o uso do método Simplex, que gera uma solução ótima.

\section{Métodos e resultados}

Este estudo foi referente ao processo de corte de etiquetas adesivas e deu sequência ao trabalho de Cechin et al. (2019) ao selecionar novos produtos de uma empresa localizada na 
Serra Gaúcha do estado do Rio Grande do Sul. Neste trabalho, foram selecionados três produtos fabricados com a mesma matéria-prima, sendo bobinas de $40 \mathrm{~cm}$ de largura.

Para atender a demanda de produção destas etiquetas, é necessário cortar as bobinas em tiras de: 200 rolos de $10 \mathrm{~cm}$ de largura, 250 rolos de $14 \mathrm{~cm}$ de largura e 350 rolos de $18 \mathrm{~cm}$ de largura. Ainda, é política da empresa manter em estoque o saldo do pedido e este estoque não deve ultrapassar a quantidade máxima de 400 rolos para a primeira peça, 500 para a segunda e 700 para a terceira. Esta regra é para evitar estoque excessivo. A Tabela 1 demonstra os possíveis padrões de corte da matéria-prima para estas três peças, e a Figura 1 representa o padrão de corte 4 .

Tabela 1 - Padrões de corte

\begin{tabular}{c|c|c|c|c|c|c}
\hline \multirow{2}{*}{$\begin{array}{c}\text { Largura da faixa } \\
\text { cortada }\end{array}$} & \multicolumn{7}{|c}{ Programação de Cortes } \\
\cline { 2 - 7 } & $\mathbf{1}$ & $\mathbf{2}$ & $\mathbf{3}$ & $\mathbf{4}$ & $\mathbf{5}$ & $\mathbf{6}$ \\
\hline $10 \mathrm{~cm}$ & 0 & 2 & 2 & 4 & 1 & 0 \\
\hline $14 \mathrm{~cm}$ & 1 & 1 & 0 & 0 & 2 & 0 \\
\hline $18 \mathrm{~cm}$ & 1 & 0 & 1 & 0 & 0 & 2 \\
\hline Desperdício $(\mathrm{cm})$ & 8 & 6 & 2 & 0 & 2 & 4 \\
\hline
\end{tabular}

Fonte: Elaborado pelos autores (2020)

Figura 1 - Padrão de corte 4

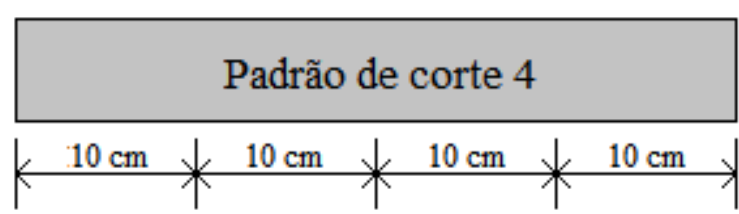

Fonte: Elaborado pelos autores (2020)

Para estas peças, não há um padrão de corte pré-determinado, a escolha dos cortes deste processo é realizada de maneira aleatória. Na última vez que houve produção destas peças, os cortes ocorreram conforme apresentado na Tabela 2.

Tabela 2 - Processo de corte

\begin{tabular}{c|c}
\hline Padrão de corte & Quantidade \\
\hline 1 & 113 \\
\hline 2 & 0 \\
\hline 3 & 65 \\
\hline 4 & 14 \\
\hline 5 & 70 \\
\hline 6 & 88 \\
\hline
\end{tabular}


De acordo com a maneira que foram cortadas as peças, conforme Tabela 2, houve um desperdício de $1.526 \mathrm{~cm}$ de matéria-prima. Com estas informações, foi desenvolvido o modelo matemático, com a função objetivo visado a minimização do desperdício de matériaprima. As variáveis $x$ de decisão do problema são referentes ao número de rolos a serem cortados de acordo com a programação de corte $i$, para $i=1,2,3,4,5$ e 6 . A função objetivo é dada pela Equação 4.

$$
F(x)=8 x_{1}+6 x_{2}+2 x_{3}+0 x_{4}+2 x_{5}+4 x_{6}
$$

Cada uma das restrições é referente a quantidade necessária de produção das peças de acordo com o pedido de venda e a política da empresa de evitar estoque em excesso. As Equações 5, 6 e 7 representam, respectivamente, a demanda da produção de adesivos de rolos de $10 \mathrm{~cm}$, de $14 \mathrm{~cm}$ e de $18 \mathrm{~cm}$ de largura.

$$
\begin{aligned}
& 0 x_{1}+2 x_{2}+2 x_{3}+4 x_{4}+0 x_{5}+0 x_{6} \geq 200 \\
& 1 x_{1}+1 x_{2}+0 x_{3}+0 x_{4}+2 x_{5}+0 x_{6} \geq 250 \\
& 1 x_{1}+0 x_{2}+1 x_{3}+4 x_{4}+0 x_{5}+2 x_{6} \geq 350
\end{aligned}
$$

Já as Equações 8, 9 e 10 são as restrições para a política da empresa em relação ao estoque máximo, respectivamente para os de rolos de $10 \mathrm{~cm}$, de $14 \mathrm{~cm}$ e de $18 \mathrm{~cm}$ de largura.

$$
\begin{aligned}
& 0 x_{1}+2 x_{2}+2 x_{3}+4 x_{4}+0 x_{5}+0 x_{6} \leq 400 \\
& 1 x_{1}+1 x_{2}+0 x_{3}+0 x_{4}+2 x_{5}+0 x_{6} \leq 500
\end{aligned}
$$




$$
1 x_{1}+0 x_{2}+1 x_{3}+4 x_{4}+0 x_{5}+2 x_{6} \leq 700
$$

Estas restrições garantem que a demanda de todos os itens seja atendida e que o excedente não supere o estoque máximo determinado pela empresa. Além disso, as restrições de decisão do problema são de não negatividade e integralidade das variáveis, utilizando Programação Linear Inteira (PLI), já que os valores são inteiros e positivos (FARIAS, 2011).

Assim, foi utilizado a técnica da PLI para calcular a otimização do problema, com a Tabela 3 apresentando os resultados obtidos.

Tabela 3 - Processo otimizado de corte

\begin{tabular}{c|c}
\hline Padrão de corte & Quantidade \\
\hline 1 & 0 \\
\hline 2 & 0 \\
\hline 3 & 38 \\
\hline 4 & 0 \\
\hline 5 & 125 \\
\hline 6 & 156 \\
\hline
\end{tabular}

Fonte: Elaborado pelos autores (2020)

Conforme apresentado na Tabela 3, o modelo determinou que a solução ótima considera 38 padrões de corte 4, 125 padrões do corte 5 e 156 do padrão 6. Com o modelo matemático desenvolvido obteve-se um a redução de desperdício de $950 \mathrm{~cm}$, ou seja, uma redução de $37,7 \%$ no desperdício de matéria-prima ao se comparar a produção atual da empresa, com a redução de $576 \mathrm{~cm}$. A partir disso, é possível sugerir a aplicação odo modelo matemático, uma vez que atende nas restrições de produção da empresa e ao mesmo tempo, visa minimizar o custo por meio da redução do desperdício de matéria-prima.

\section{Conclusões}

Este artigo analisou a importância da PL aplicado na redução da perda de matéria-prima no processo de corte, em uma empresa que fabrica etiquetas adesivas, presente na Serra Gaúcha, do estado do Rio Grande do Sul. Para aprimorar este período produtivo, foi recomendado uma PL para com uma modelação matemática eficaz para calcular uma solução ótima, minimizando a perda do material. 
A comparação do valor obtido com a otimização foi feita com os valores aplicados pela empresa, onde é possível verificar que a mesma não utiliza a combinação ideal para o corte. Assim, foi comprovado uma possível redução de $576 \mathrm{~cm}$ de material, o que representa 37,7\% a menos de desperdício, permitindo, inclusive, uma redução de custos do produto.

Com os resultados obtidos, julga-se a grande importância da aplicação de técnicas de PO, como a PL, que auxiliam os gestores de organizações na tomada de decisão de maneira racional, focados em desempenho e performance.

Para aplicação deste modelo, destaca-se como fundamental um trabalho próximo entre programação da produção e planejamento. Isso deve-se a necessidade de o modelo matemático ser criado de forma a representar o interesse da organização.

A aplicação de um modelo matemático, via PL, para minimização do desperdício de matériaprima em problema de corte é considerando economicamente relevante, uma vez que o custo total do produto final apresenta dependência direta deste parâmetro. Os valores apresentados de redução aqui comprovam a possibilidade de utilização modelo, uma vez que atendeu todas as restrições. Desta forma, pode-se considerar que os objetivos foram atingidos. Como limitação deste modelo, pode-se considerar que dados de planejamento estratégico ou mesmo redução direta do custo de estoque não foram considerados.

\section{REFERÊNCIAS}

ALEM JUNIOR. Douglas José. O problema de corte de estoque com demanda estocástica. 2007, 98 f. Dissertação (Mestrado em Ciências da Computação e Matemática Computacional) - Universidade de São Paulo, São Carlos, 2007.

ALMEIDA, Mailton Rego. Programação linear: Uma aplicação ao problema de compras de um supermercado da cidade de Macaúbas-BA. 2018, 59 f. (Graduação em Matemática) Universidade Estadual do Sudoeste da Bahia, Macaúbas, 2018.

ANDRADE, Eduardo Leopoldino. Introdução à pesquisa operacional: métodos e modelos para análise de decisões. 5. ed. Rio de Janeiro: LTC, 2015.

ASSIS, Nícolas Samuel. O problema de corte de estoque bidimensional: geração de padrões de corte 2-estágios restritos. 2019, 98 f. Dissertação (Mestrado em Matemática) Universidade Estadual Paulista, São José do Rio Preto, 2019.

CARTER, Michael W.; PRICE, Camille C.. Operations research: a practical introduction. Boca Raton: CRC Press, 2001. 
CECHIN, Rafaela B.; BIASUZ, Rodrigo; ORLANDIN, Bruna; TOME, Fernanda; CORSO, Leandro. Otimização de corte para etiquetas adesivas: um estudo de caso. Simpósio de Engenharia, Gestão e Inovação, Águas de Lindóia - SP, 2019.

FARIAS, Poliane C. de. Problema de corte de estoque unidimensional com reaproveitamento de sobras: abordagem de resolução por meio de uma técnica de geração de colunas. 2011, 108 f. Dissertação (Mestrado em Matemática Aplicada e Computacional) Universidade Estadual de Londrina, Londrina, 2011.

GHIDINI, Carla T. L. da S. Otimização de processos acoplados: programação da produção e corte de estoque. 2009, 151 f. Tese (Doutorado em Ciências da Computação e Matemática Computacional) - Universidade de São Paulo, São Carlos, 2009.

GOMES, Herbert M.; CORSO, Leandro L. A Hybrid Method for Truss Mass

Minimization considering Uncertainties. Mathematical Problems in Engineering, 2017.

HILLIER, Frederick S.; LIEBERMAN, Gerald J.. Introdução à pesquisa operacional. 9. ed. Porto Alegre: AMGH, 2013.

LOESCH, Cláudio; HEIN, Nelson. Pesquisa operacional: fundamentos e modelos. Saraiva, 2009.

LONGARAY, André Andrade. Introdução à pesquisa operacional. São Paulo: Saraiva, 2013.

MOREIRA, Daniel Augusto. Pesquisa operacional: curso introdutório. São Paulo: Thomson Learning, 2007.

PERINI, Gustavo; LUCIANO, Marcos Alexandre; CORSO, Leandro Luis. An Analysis of the Topological Optimization from the Point of View of Seven Wastes. IEEE Latin America Transactions, v. 14, n. 2, p. 858-862, 2016.

POLDI, Kelly Cristina. Algumas extensões do problema de corte de estoque. 2003, $141 \mathrm{f}$. Dissertação (Mestrado em Ciências da Computação e Matemática Computacional) Universidade de São Paulo, São Carlos, 2003.

POLDI, Kelly Cristina; ARENALES, Marcos Nereu. O problema de corte de estoque unidimensional multiperíodo. Pesquisa Operacional, v. 30, n. 1, p. 153-174, 2010.

SILVA, E. M.; SILVA, E. M.; GONÇALVES, V.; MUROLO, A. C. Pesquisa Operacional para os cursos de administração e engenharia: programação linear: simulação. 5 ed. São Paulo: Atlas, 2017. 